\title{
ON THE STRUCTURE JACOBI OPERATOR AND RICCI TENSOR OF REAL HYPERSURFACES IN NONFLAT COMPLEX SPACE FORMS
}

\author{
SoO JIN KIM
}

\begin{abstract}
It is known that there are no real hypersurfaces with parallel structure Jacobi operator $R_{\xi}$ (cf.[16], [17]). In this paper we investigate real hypersurfaces in a nonflat complex space form using some conditions of the structure Jacobi operator $R_{\xi}$ which are weaker than $\nabla R_{\xi}=0$. Under further condition $S \phi=\phi S$ for the Ricci tensor $S$ we characterize Hopf hypersurfaces in a complex space form.
\end{abstract}

\section{Introduction}

A complex $n$-dimensional Kähler manifold of constant holomorphic sectional curvature $4 c$ is called a complex space form, which is denoted by $M_{n}(c)$. As is well-known, a complete and simply connected complex space form is complex analytically isometric to a complex projective space $P_{n} \mathbb{C}$, a complex Euclidean space $\mathbb{C}_{n}$ or a complex hyperbolic space $H_{n} \mathbb{C}$, according as $c>0, c=0$ or $c<0$.

In this paper we consider a real hypersurface $M$ in a complex space form $M_{n}(c), c \neq 0$. Then $M$ has an almost contact metric structure $(\phi, g, \xi, \eta)$ induced from the Kähler metric and complex structure $J$ on $M_{n}(c)$. The structure vector field $\xi$ is said to be principal if $A \xi=\alpha \xi$ is satisfied, where $A$ is the shape operator of $M$ and $\alpha=\eta(A \xi)$. In this case, it is well known that $\alpha$ is a locally constant ([13]) and that $M$ is called a Hopf hypersurface.

Typical examples of Hopf hypersurfaces in $P_{n} \mathbb{C}$ are homogeneous ones, namely those real hypersurfaces are given as orbits under subgroup of the projective unitary group $P U(n+1)$. Takagi [18], [19] completely classified such hypersurfaces as six model spaces which are said to be of type $A_{1}, A_{2}, B, C, D$ and $E$. Also Berndt ([1]) showed that all Hopf real

Received November 19, 2010. Accepted December 06, 2010.

2000 Mathematics Subject Classification. Primary 53C40, Secondary 53C15.

Keywords and phrases. Hopf hypersurface, structure Jacobi operator, Ricci tensor. 
hypersurfaces with constant principal curvature in a complex hyperbolic space $H_{n} \mathbb{C}$ are realized as the tubes of constant radius over certain submanifolds. Nowadays in $H_{n} \mathbb{C}$ they are said to be of type $A_{0}, A_{1}, A_{2}$ and $B$. In 2007, Berndt and Tamaru [2] classified all homogeneous real hypersurfaces in $H_{n} \mathbb{C}$. Real hypersurfaces of each type in $M_{n}(c), c \neq 0$ have been described in detail by Niebergall and Ryan ([15]).

On the other hand, the structure Jacobi operator $R_{\xi}=R(\cdot, \xi) \xi$ for the Riemannian curvature operator $R$ has a fundamental role in contact geometry. Cho and Ki started the study on real hypersurfaces in a complex space form by using the operator $R_{\xi}$ in [3], [4] and [5]. It is well known that there are no real hypersurfaces with parallel structure Jacobi operator $\nabla R_{\xi}=0$ in $M_{n}(c), c \neq 0$ ([16], [17]).

Motivated by results mentioned above, it is natural to investigate real hypersurfaces in a complex space form by using some conditions (on the derivative of $R_{\xi}$ ) which are weaker than $\nabla R_{\xi}=0$. From this point of views, some works have studied real hypersurfaces with $\nabla_{\xi} R_{\xi}=0$ or $\nabla_{\phi \nabla_{\xi} \xi} R_{\xi}=0$, and given some results on the classification of real hypersurfaces in a complex space form $([6] \sim[12],[14])$. Two of them we introduce the following without proof.

Theorem 1.1 (Ki, Kurihara, Nagai and Takagi [9]). Let $M$ be a real hypersurface in a complex space form $M_{n}(c), c \neq 0$ whose Ricci tensor commutes with $R_{\xi}$. Then $M$ satisfies $\nabla_{\xi} R_{\xi}=0$ if and if only $M$ is locally congruent to one of the following:

(1) In case that $M_{n}(c)=P_{n} \mathbb{C}$ with $\eta(A \xi) \neq 0$, $r \neq \pi / 4$

$\left(A_{1}\right)$ a geodesic hypersphere of radius $r$, where $0<r<\pi / 2$ and

$\left(A_{2}\right)$ a tube of radius $r$ over a totally geodesic $P_{k} \mathbb{C}(1 \leq k \leq n-2)$, where $0<r<\pi / 2$ and $r \neq \pi / 4$.

(2) In case $M_{n}(c)=H_{n} \mathbb{C}$,

$\left(A_{0}\right)$ a horosphere,

$\left(A_{1}\right)$ a geodesic hypersphere or a tube over a complex hyperbolic hyperplane $H_{n-1} \mathbb{C}$,

$\left(A_{2}\right)$ a tube over a totally geodesic $H_{n} \mathbb{C}(1 \leq n \leq n-2)$.

Theorem 1.2 (Ki and Kurihara [7]). Let $M$ be a real hypersurface in a nonflat complex space form which satisfies $\nabla_{\phi \nabla_{\xi} \xi} R_{\xi}=0$. M holds $\nabla_{\xi} R_{\xi}=0$ if and only if $M$ is locally congruent to one of (1) and (2) stated in Theorem 1.1. 
Let us denote the Ricci tensor of type $(1,1)$ by $S$. If we pay a particular attention to the fact that for each Hopf hypersurface $M$ in $M_{n}(c)$, $c \neq 0$, then $S \xi=g(S \xi, \xi) \xi$ is satisfied. If $S \phi=\phi S$ holds on real hypersurfaces in $M_{n}(c)$, then we have $S \xi=g(S \xi, \xi) \xi$. The classification of such real hypersurfaces is still open problem. Recently we have the following:

Theorem 1.3 (Kim and $\mathbf{K i}[\mathbf{1 4}])$. Let $M$ be a real hypersurface in $M_{n}(c), c \neq 0$, which satisfies $\nabla_{\xi} R_{\xi}=0$ and at the same time $\phi S=S \phi$. Then $M$ is a Hopf hypersurface. Further, $M$ is locally congruent to one of (1) and (2) stated in Theorem 1.1.

Theorem 1.4 (Ki and Nagai [11]). Let $M$ be a real hypersurface in a complex projective space $P_{n} \mathbb{C}$ which satisfies $R_{\xi} S=S R_{\xi}$ and $\nabla_{\phi \nabla_{\xi} \xi} S=0$. If $g(S \xi, \xi)=$ const., then $M$ is a Hopf hypersurface.

In this paper, we investigate real hypersurface satisfying $\phi S=S \phi$ and at the same time $\nabla_{\phi \nabla_{\xi} \xi} R_{\xi}=0$ in a complex space form. The main purpose of the present paper is to establish Main Theorem stated in section 5 .

All manifolds in the present paper are assumed to be connected and of $C^{\infty}$ and real hypersurfaces supposed to be orientable.

\section{Preliminaries}

Let $M$ be a real hypersurface immersed in a complex space form $M_{n}(c)$ with parallel almost complex structure $J$, and constant holomorphic sectional curvature $4 c$ and $N$ be a unit normal vector field on $M$. By $\tilde{\nabla}$ we denote the Levi-Civita connection with respect to the Fubinistudy metric $\tilde{g}$ of $M_{n}(c)$. Then the Gauss and Weingraten formulas are given respectively by

$$
\tilde{\nabla}_{X} Y=\nabla_{X} Y+g(A X, Y) N, \quad \tilde{\nabla}_{X} N=-A X
$$

for any vector fields $X$ and $Y$ on $M$, where $\nabla$ and $g$ denote the Riemannian connection and the Riemannian metric tensor induced from $\tilde{g}$ respectively, and $A$ denotes the shape operator in the direction of $N$.

For any vector field $X$ tangent to $M$, we put

$$
J X=\phi X+\eta(X) N, \quad J N=-\xi .
$$

We call $\xi$ the structure vector field and its flow also denoted by the same $\xi$. Then we may see that the aggregate $(\phi, \xi, \eta, g)$ is an almost 
contact metric structure on $M$. From the fact $\tilde{\nabla} J=0$, and by using the Gauss and Weingarten formulas, we have

$$
\begin{gathered}
\nabla_{X} \xi=\phi A X \\
\left(\nabla_{X} \phi\right) Y=\eta(Y) A X-g(A X, Y) \xi .
\end{gathered}
$$

Since we consider that the ambient manifold is of constant holomorphic sectional curvature $4 c$, we have the following Gauss and Codazzi equations respectively.

$$
\begin{gathered}
R(X, Y) Z=c\{g(Y, Z) X-g(X, Z) Y+g(\phi Y, Z) \phi X \\
-g(\phi X, Z) \phi Y-2 g(\phi X, Y) \phi Z\} \\
+g(A Y, Z) A X-g(A X, Z) A Y \\
\left(\nabla_{X} A\right) Y-\left(\nabla_{Y} A\right) X=c\{\eta(X) \phi Y-\eta(Y) \phi X-2 g(\phi X, Y) \xi\}
\end{gathered}
$$

for any tangent vector fields $X, Y$ and $Z$ on $M$, where $R$ denote the Riemannian curvature tensor of $M$.

Now let us denote by $\alpha=\eta(A \xi), \beta=\eta\left(A^{2} \xi\right)$ and $h=\operatorname{Tr} A$, and $\nabla f$ the gradient vector field of a function $f$ defined on $M$.

If we put $U=\nabla_{\xi} \xi$, then $U$ is orthogonal to $\xi$. Thus, it is, using (2.1), seen that

$$
\phi U=-A \xi+\alpha \xi,
$$

which leads to $g(U, U)=\beta-\alpha^{2}$. We easily see that $\xi$ is a principal curvature vector field, that is $A \xi=\alpha \xi$, if and only if $\beta-\alpha^{2}=0$. In this case, $M$ is called a Hopf hypersurface in $M_{n}(c)$. If we put

$$
A \xi=\alpha \xi+\mu W,
$$

where $W$ is a unit vector field orthogonal to $\xi$. Then we get $U=\mu \phi W$, which tells us that $W$ is also orthogonal to $U$. Further, we have

$$
\mu^{2}=\beta-\alpha^{2} .
$$

Using (2.1) and (2.6), we see that

$$
\begin{gathered}
\mu g\left(\nabla_{X} W, \xi\right)=g(A U, X), \\
g\left(\nabla_{X} \xi, U\right)=\mu g(A W, X)
\end{gathered}
$$

for any vector field $X$ on $M$. 
Differentiating (2.5) covariantly along $M$ and making use of (2.1) and (2.2), we find

$$
\left(\nabla_{X} A\right) \xi=-\phi \nabla_{X} U+g(A U+\nabla \alpha, X) \xi-A \phi A X+\alpha \phi A X
$$

which enables us to obtain

$$
\left(\nabla_{\xi} A\right) \xi=2 A U+\nabla \alpha
$$

where we have used (2.4). From (2.1) and (2.10), we verify that

$$
\nabla_{\xi} U=3 \phi A U+\alpha A \xi-\beta \xi+\phi \nabla \alpha .
$$

From the Gauss equation (2.3) the structure Jacobi operator $R_{\xi}$ is given by

$$
R_{\xi} X=R(X, \xi) \xi=c\{X-\eta(X) \xi\}+\alpha A X-\eta(A X) A \xi
$$

for any vector field $X$ on $M$.

We set $\Omega=\{p \in M \mid \mu(p) \neq 0\}$, and suppose that $\Omega \neq \varnothing$, that is $\xi$ is not principal curvature vector on $M$. Hereafter, unless otherwise stated, we continue our discussions on the open set $\Omega$ of $M$.

Differentiating (2.13) covariantly along $\Omega$, we find

$$
\begin{aligned}
& =g\left(\nabla_{X}\left(R_{\xi} Y\right)-R_{\xi}\left(\nabla_{X} Y\right), Z\right) \\
& =-c\left\{\eta(Z) g\left(\nabla_{X} \xi, Y\right)+\eta(Y) g\left(\nabla_{X} \xi, Z\right)\right\}+(X \alpha) g(A Y, Z) \\
& +\alpha g\left(\left(\nabla_{X} A\right) Y, Z\right)-\eta(A Z)\left\{g\left(\left(\nabla_{X} A\right) \xi, Y\right)+g(A \phi A X, Y)\right\} \\
& \quad-\eta(A Y)\left\{g\left(\left(\nabla_{X} A\right) \xi, Z\right)+g(A \phi A X, Z)\right\} .
\end{aligned}
$$

If we put $X=W$ and $Y=\xi$ in (2.14), then we get

$$
\begin{aligned}
& g\left(\left(\nabla_{W} R_{\xi}\right) \xi, Z\right) \\
= & -c g(\phi A W, Z)+(W \alpha) g(A \xi, Z)-\eta(A Z) g\left(\left(\nabla_{W} A\right) \xi, \xi\right) \\
& -\eta(A Z) g(A \phi A W, Z)-\alpha g(A \phi A W, Z),
\end{aligned}
$$

or using (2.11)

$$
g\left(\left(\nabla_{W} R_{\xi}\right) \xi, Z\right)=-\alpha g(A \phi A W, Z)-c g(\phi A W, Z)-g(A U, W) \eta(A Z) .
$$

\section{Real hypersurfaces satisfying $S \phi=\phi S$}

In the following we shall denote the Ricci tensor of type $(1,1)$ by $S$. Then it follows from (2.3) that

$$
S=c\{(2 n+1) I-3 \eta \otimes \xi\}+h A-A^{2},
$$


where $I$ is an identity map, which implies

$$
S \xi=2 c(n-1) \xi+h A \xi-A^{2} \xi .
$$

Now, suppose that $S \phi=\phi S$ is satisfied on a real hypersurface $M$ of $M_{n}(c), c \neq 0$. Then we have from (3.1) $A^{2} \phi-\phi A^{2}=h(A \phi-\phi A)$, which enables us to obtain $\phi\left(A^{2} \xi-h A \xi\right)=0$. Because of properties of the almost contact metric structure $(\phi, g, \xi, \eta)$, it follows, using this, that

$$
A^{2} \xi=h A \xi+(\beta-h \alpha) \xi .
$$

Combining (2.6) to this, we verify that

$$
A W=\mu \xi+(h-\alpha) W
$$

and hence

$$
A^{2} W=h A W+(\beta-h \alpha) W
$$

because of $\mu \neq 0$. Differentiating (3.4) covariantly along $\Omega$, we find

(3.6) $\left(\nabla_{X} A\right) W+A \nabla_{X} W=(X \mu) \xi+\mu \nabla_{X} \xi+X(h-\alpha) W+(h-\alpha) \nabla_{X} W$.

If we take an inner product with $W$ in the last equation, then we find

$$
g\left(\left(\nabla_{X} A\right) W, W\right)=-2 g(A U, X)+X h-X \alpha
$$

since $W$ is a unit vector field orthogonal to $\xi$. We also obtain by applying $\xi$ to $(3.6)$,

$$
\mu g\left(\left(\nabla_{X} A\right) W, \xi\right)=(h-2 \alpha) g(A U, X)+\mu(X \mu),
$$

where we have used (2.8), which together with the Codazzi equation (2.4) implies that

$$
\mu\left(\nabla_{\xi} A\right) W=(h-2 \alpha) A U-c U+\mu \nabla \mu .
$$

Replacing $X$ by $\mu \xi$ in (3.6) and taking account of (3.8), we get

$$
\begin{gathered}
(h-2 \alpha) A U-c U+\mu \nabla \mu+\mu\left\{A \nabla_{\xi} W-(h-\alpha) \nabla_{\xi} W\right\} \\
=\mu(\xi \mu) \xi+\mu^{2} U+\mu(\xi h-\xi \alpha) W .
\end{gathered}
$$

By the way, from $\phi U=-\mu W$ we see, using (2.2,) that

$$
g(A U, X) \xi-\phi \nabla_{X} U=(X \mu) W+\mu \nabla_{X} W .
$$

Putting $X=\xi$ in this and making use of (2.5) and (2.12), we obtain

$$
\mu \nabla_{\xi} W=3 A U-\alpha U+\nabla \alpha-(\xi \alpha) \xi-(\xi \mu) W .
$$

Substituting this into (3.9), we have

$$
\begin{gathered}
3 A^{2} U-2 h A U+A \nabla \alpha+\frac{1}{2} \nabla \beta-h \nabla \alpha+(\alpha h-\beta-c) U \\
=2 \mu(W \alpha) \xi+\mu(\xi h) W-(h-2 \alpha)(\xi \alpha) \xi,
\end{gathered}
$$


where we have used (2.7). Taking an inner product $\xi$ to this and using (2.6) and (2.7), we find

$$
\xi \mu=W \alpha .
$$

From (3.7) and (3.8), it is seen that

$$
W \mu=\xi h-\xi \alpha \text {. }
$$

Differentiating (3.3) covariantly and making use of (2.1), we find

$$
\begin{gathered}
\left(\nabla_{X} A\right) A \xi+A\left(\nabla_{X} A\right) \xi+A^{2} \phi A X-h A \phi A X \\
=(X h) A \xi+h\left(\nabla_{X} A\right) \xi+X(\beta-h \alpha) \xi+(\beta-h \alpha) \phi A X,
\end{gathered}
$$

which together with the Codazzi equation (2.4) implies that

$$
\begin{gathered}
c\{u(Y) \eta(X)-u(X) \eta(Y)\}+2 c(h-\alpha) g(\phi X, Y)-g\left(A^{2} \phi A X, Y\right) \\
+g\left(A^{2} \phi A Y, X\right)+2 h g(\phi A X, Y)-(\beta-h \alpha)\{g(\phi A Y, X)-g(\phi A X, Y)\} \\
=g\left(A Y,\left(\nabla_{X} A\right) \xi\right)-g\left(A X,\left(\nabla_{Y} A\right) \xi\right)+(Y h) g(A \xi, X)-(X h) g(A \xi, Y) \\
+Y(\beta-h \alpha) \eta(X)-X(\beta-h \alpha) \eta(Y),
\end{gathered}
$$

where we have defined a 1 -form $u$ by $u(X)=g(U, X)$ for any vector field $X$. If we replace $X$ by $\mu W$ to the both sides of the last equation and make use of (2.4), (2.11), (3.4), (3.5) and (3.8), then we get

$$
\begin{aligned}
& (3 \alpha-2 h) A^{2} U+2\left(h^{2}+\beta-2 h \alpha+c\right) A U+(h-\alpha)(\beta-h \alpha-2 c) U \\
= & \mu A \nabla \mu+(\alpha h-\beta) \nabla \alpha-\frac{1}{2}(h-\alpha) \nabla \beta+\mu^{2} \nabla h-\mu(W h) A \xi \\
& -\mu W(\beta-h \alpha) \xi .
\end{aligned}
$$

Applying by $U$ or $W$ in (3.1), we have respectively

$$
\begin{aligned}
& S U=(2 n+1) c U+h A U-A^{2} U, \\
& S W=(2 n+1) c W+(h \alpha-\beta) W
\end{aligned}
$$

by virtue of (3.5). If we take an inner product $U$ to $S \phi-\phi S=0$ and make use of the last two equations, then we obtain

$$
-\mu S W+(2 n+1) c \mu W-h \phi A U+\phi A^{2} U=0,
$$

or equivalently $(\beta-h \alpha) \mu W-h \phi A U+\phi A^{2} U=0$. Thus, it follows that

$$
A^{2} U=h A U+(\beta-h \alpha) U .
$$


4. Real hypersurfaces satisfying $S \phi=\phi S$ and $\nabla_{\phi U} R_{\xi}=0$

We will continue our arguments on $\Omega$ under the assumptions $S \phi=\phi S$ and at the same time $\nabla_{\phi \nabla_{\xi} \xi} R_{\xi}=0$. Then the second hypothesis implies that $\nabla_{W} R_{\xi}=0$ because of $(2.5)$ and (2.6). So see from (2.15) that

$$
\alpha A \phi A W+c \phi A W+g(A U, W) \xi=0 .
$$

Since the first assumption implies (3.4). So we obtain $g(A W, U)=0$. Therefore (4.1) reformed as

$$
\alpha A \phi A W+c \phi A W=0 .
$$

Combining this to (3.4), we see that

$$
\lambda(\alpha A U+c U)=0 .
$$

because of (2.5), where we have put $\lambda=h-\alpha$.

We notice here that the following:

Remark 1. $\alpha \neq 0$ on $\Omega$.

In fact, if not, then we get $\alpha=0$. So we verify, using (4.3), that $\lambda=0$ and hence $h=0$ on this subset. Consequently (3.11), (3.14) and (3.15) are reduced respectively to

$$
\begin{gathered}
3 A^{2} U+\frac{1}{2} \nabla \beta-(\beta+c) U=0, \\
2(\beta+c) A U=\frac{1}{2} A \nabla \beta-\mu(W \beta) \xi, \\
A^{2} U=\beta U
\end{gathered}
$$

on the subset, where we have used (2.7). Combining these we obtain $4 \beta A U+\mu(W \beta) \xi=0$, which together with (2.6) yields $W \beta=0$ and thus $A U=0$ on the set because $\beta \neq 0$ on $\Omega$. From the equation $A^{2} U=\beta U$ we have $U=0$, a contradiction. Therefore $\alpha \neq 0$ is proved on $\Omega$.

Putting $X=W$ in (2.14) and using (4.2) and Remark 1, we find

$$
\begin{aligned}
\alpha\left(\nabla_{W} A\right) X= & -(W \alpha) A X+g(A \xi, X)\left(\nabla_{W} A\right) \xi+g\left(\left(\nabla_{W} A\right) \xi, X\right) A \xi \\
& -\frac{c}{\alpha}\{w(X) \phi A W+g(\phi A W, X) W\},
\end{aligned}
$$

where we have defined a 1-form $w$ by $w(X)=g(W, X)$ for any vector field $X$, which connected to (3.4) and (3.8) gives

$$
\alpha\left(\nabla_{W} A\right) X=-(W \alpha) A X-\frac{c}{\alpha} \lambda(w(X) U+u(X) W)
$$




$$
\begin{aligned}
& +\frac{1}{\mu}\{(\lambda-\alpha) A U-2 c U+\mu \nabla \mu\} g(A \xi, X) \\
& +\frac{1}{\mu} g((\lambda-\alpha) A U-2 c U+\mu \nabla \mu, X) A \xi
\end{aligned}
$$

If we put $X=W$ in (4.4) and take account of (2.7), (3.7) and (3.13), then we obtain

$$
\text { (4.5) } \frac{1}{2} \alpha \nabla \beta-\alpha^{2} \nabla h=c(h+\alpha) U-\alpha h A U+\alpha(W \alpha) A W-\alpha(\xi h-\xi \alpha) A \xi \text {. }
$$

First of all, we shall prove the following lemma, which will be used later.

Lemma 1. $\alpha A U+c U=0$ on $\Omega$.

Proof. We suppose that $\alpha A U+c U \neq 0$ on $\Omega$, and then we restrict the arguments on such a place. From (4.3) we then have $\lambda=0$, that is, $h-\alpha=0$. Accordingly (3.4) is reduced to $A W=\mu \xi$. So (3.14) turns out to be

$$
\mu(W \alpha) A \xi=\mu A \nabla \mu-\alpha A^{2} U-2\left(\mu^{2}+c\right) A U,
$$

where we have used (2.7) and (3.13). In the same way we see from (4.5) that

$$
\mu \nabla \mu=2 c U-\alpha A U+\mu(W \alpha) \xi
$$

because of (2.7) and Remark 1, which implies

$$
\mu A \nabla \mu=2 c A U-\alpha A^{2} U+\mu(W \alpha) A \xi .
$$

Thus, (4.6) together with this yields

$$
\alpha A^{2} U+\mu^{2} A U=0,
$$

or using (2.7) and (3.15) $\beta A U+\alpha \mu^{2} U=0$. From this we have $\beta A^{2} U+$ $\alpha \mu^{2} A U=0$. Hence we have $A^{2} U=0$ by virtue of (4.7). So we obtain $A U=0$ and therefore $U=0$, a contradiction. This completes the proof.

Using (3.15) and Lemma 1, we have

$$
\alpha^{2}(\beta-h \alpha)=c(h \alpha+c) .
$$

By Lemma 1, (4.5) turns out to be

$$
\frac{1}{2} \alpha^{2} \nabla \beta=\alpha^{2} \nabla h+c(\alpha+2 h) U+\alpha(W \alpha) A W-\alpha(\xi h-\xi \alpha) A \xi .
$$

Applying (4.9) by $W$ and making use of $(2.7)$ and $(3.13$,$) we find$

$$
W \beta=\alpha(W h)+h(W \alpha) .
$$


Because of Lemma 1 and Remark 1, we can write (4.4) as

$$
\begin{aligned}
& \alpha\left(\nabla_{W} A\right) X=-(W \alpha) A X-\frac{c \lambda}{\alpha}\{u(X) W+w(X) U\} \\
& +g\left(\nabla \mu-\frac{c h}{\alpha \mu} U, X\right) A \xi+g(A \xi, X)\left(\nabla \mu-\frac{c h}{\alpha \mu} U\right) .
\end{aligned}
$$

By putting $X=U$ in this, and making use of Lemma 1, we obtain

$$
\alpha\left(\nabla_{W} A\right) U=(U \mu) A \xi+\frac{c}{\alpha}\left((W \alpha) U-\lambda \mu^{2} W-h \mu A \xi\right) .
$$

On the other hand, if we take an inner product $U$ to (3.6) and make use of (2.4) and Lemma 1, then we obtain

$$
(\alpha \lambda+c) g\left(\nabla_{X} W, U\right)=\alpha g\left(\left(\nabla_{W} A\right) X, U\right)+c \alpha \mu \eta(X)-\alpha \mu^{2} g(A W, X),
$$

which together with (4.12) implies that

$$
\begin{aligned}
& (\alpha \lambda+c) g\left(\nabla_{X} W, U\right)=\frac{c}{\alpha} g\left((W \alpha) U-\lambda \mu^{2} W-\mu h A \xi, X\right) \\
& +(U \mu) g(A \xi, X)+\alpha \mu \eta(X)-\alpha \mu^{2} g(A W, X) .
\end{aligned}
$$

Putting $X=U$ in this and using (3.4), we have

$$
(\alpha \lambda+c) g\left(\nabla_{U} W, U\right)=\frac{c}{\alpha} \mu^{2}(W \alpha) .
$$

Now, if we differentiate $\alpha A U+c U=0$ covariantly, and use itself again, then we get

$$
-\frac{c}{\alpha}(X \alpha) U+\alpha\left(\nabla_{X} A\right) U+\alpha A \nabla_{X} U+c \nabla_{X} U=0,
$$

which together with the Codazzi equation (2.4) and (2.5) yields

$$
\begin{aligned}
& \frac{c}{\alpha}\{(Y \alpha) u(X)-(X \alpha) u(Y)\}+c \alpha \mu\{\eta(X) w(Y)-\eta(Y) w(X)\} \\
& +\alpha\left\{g\left(A \nabla_{X} U, Y\right)-g\left(A \nabla_{Y} U, X\right)\right\}+c d u(X, Y)=0,
\end{aligned}
$$

where $d u$ is the exterior derivative of a 1-form $u$ given by

$$
d u(X, Y)=X(u(Y))-Y(u(X))-u([X, Y]) .
$$

Replacing $X$ by $\xi$ in the last equation and taking account of (2.6) and (2.9), we obtain

$$
\begin{aligned}
\alpha \mu g\left(W, \nabla_{X} U\right)=- & \frac{c}{\alpha}(\xi \alpha) u(X)+c \alpha \mu w(X)+\mu\left(\alpha^{2}+c\right) g(A W, X) \\
& +g\left(\alpha A \nabla_{\xi} U+c \nabla_{\xi} U, X\right) .
\end{aligned}
$$

If we put $X=U$ in this and make use of $\alpha A U+c U=0$, then we have $\alpha^{2} g\left(\nabla_{U} W, U\right)=c \mu(\xi \alpha)$, which together with (4.14) implies that

$$
\alpha \mu(W \alpha)=(\alpha \lambda+c) \xi \alpha .
$$


Differentiating (4.8) along $\Omega$ and using itself again, we find

$$
\alpha^{2} \nabla \beta=-\frac{2 c}{\alpha}(h \alpha+c) \nabla \alpha+\left(\alpha^{2}+c\right)(\alpha \nabla h+h \nabla \alpha) .
$$

If we take an inner product $W$ to this and make use of (4.10), then we obtain

$$
\alpha(W \beta)=2(h \alpha+c) W \alpha
$$

Finally we prove

Lemma 2. $\xi \alpha=0, \xi h=0, \xi \beta=0$ and $W \alpha=0$ on $\Omega$.

Proof. It is verify, using (2.7), that we have from (3.13) $\frac{1}{2} W \beta=$ $\mu(\xi \lambda)+\alpha(W \alpha)$, which together with (4.18) implies that $(\alpha \lambda+c) W \alpha=$ $\mu \alpha(\xi \lambda)$, where we have used $\lambda=h-\alpha$. Thus, it follows that

$$
(\alpha \lambda+c) W \alpha=\mu \alpha(\xi h)-\mu \alpha(\xi \alpha) .
$$

On the other hand, applying (4.17) by $\xi$ we obtain,

$$
\alpha^{2}(\xi \beta)=-\frac{2 c}{\alpha}(h \alpha+c) \xi \alpha+\left(\alpha^{2}+c\right)(\alpha \xi h+h \xi \alpha) .
$$

By the way, (2.7) and (3.12) implies that $\alpha(\xi \beta)=2 \mu \alpha(W \alpha)+$ $2 \alpha^{2}(\xi \alpha)$, which connected to (4.16) gives $\alpha(\xi \beta)=2(\alpha h+c) \xi \alpha$. Substituting the last equation into (4.20), we find

$$
\left(\alpha^{2}+c\right)\left\{\alpha^{2}(\xi h)-(h \alpha+2 c) \xi \alpha\right\}=0 .
$$

Let $\Omega_{0}$ be the set of points such that $(\xi \alpha)_{p} \neq 0, p \in \Omega$ and suppose that $\Omega_{0}$ be nonempty. Then we have on $\Omega_{0} \alpha^{2}(\xi h)-(h \alpha+2 c) \xi \alpha=0$.

This, together with (4.19) implies that $\alpha(\alpha \lambda+c) W \alpha=\mu(\alpha \lambda+2 c) \xi \alpha$. Accordingly we have from this and (4.16) on $\Omega_{0}$.

$$
(\alpha \lambda+c)^{2}=\mu^{2}(\alpha \lambda+2 c) .
$$

Since we have $\alpha^{2} \mu^{2}=\alpha^{3} \lambda+c\left(\alpha \lambda+\alpha^{2}+c\right)$ by virtue of (4.8), the last relationship reformed as $(\alpha \lambda+c)\left(\alpha \lambda+\alpha^{2}+2 c\right)=0$ on $\Omega_{0}$. If $\alpha \lambda+c=0$, then $\alpha h+c=\alpha^{2}$. Thus, (4.8) implies $\beta-h \alpha=c$ and hence $\beta=\alpha^{2}$ i.e $\mu=0$, it cannot occur on $\Omega$, a contradiction. Therefore we have $\alpha \lambda+\alpha^{2}+2 c=0$ on $\Omega_{0}$. So we have $\alpha h+2 c=0$ and therefore $\alpha(W h)+h(W \alpha)=0$ on $\Omega_{0}$, which together with (4.10) yields $W \beta=0$. Thus, (4.18) gives $W \alpha=0$ and hence $\xi \alpha=0$ on $\Omega_{0}$ by virtue of (4.16). Thus $\xi \alpha=0$ every where on $\Omega$ is proved. Therefore we have $W \alpha=0$, $\xi h=0$ and $\xi \beta=0$ because of (4.16), (4.19) and (4.20) respectively. This completes the proof of Lemma2. 


\section{Main theorem}

We will continue our arguments on $\Omega$ under the hypotheses as those stated in section 4 . We prove

Theorem. Let $M$ be a real hypersurface with almost contact metric structure $(\phi, g, \xi, \eta)$ in a complex space form which satisfies $\phi S=S \phi$ and at the same time $\nabla_{\phi \nabla_{\xi} \xi} R_{\xi}=0$. Then $M$ is a Hopf hypersurface, where $S$ denotes the Ricci tensor of $M$.

Proof. Because of Lemma 2. we can write (4.9) as

$$
\frac{1}{2} \alpha \nabla \beta=\alpha^{2} \nabla h+c(\alpha+2 h) U,
$$

which together with (4.17) implies that

$$
\left(\operatorname{ch} \alpha-h \alpha^{3}+2 c^{2}\right) \nabla \alpha+\alpha^{2}\left(\alpha^{2}-c\right) \nabla h=-2 c \alpha^{2}(2 h+\alpha) U .
$$

This is rewritten as

$$
\left(c h \alpha-h \alpha^{3}+2 c^{2}\right)(Y \alpha)+\alpha^{2}\left(\alpha^{2}-c\right)(Y h)+2 c \alpha^{2}(2 h+\alpha) u(Y)=0
$$

for any vector field $Y$. Differentiating this with respect to a vector field $X$, and taking the skew-symmetric parts for $X$ and $Y$, we eventually have

$3\left(c-\alpha^{2}\right)\{(X h)(Y \alpha)-(Y h)(X \alpha)\}+2 c(4 h+3 \alpha)\{(X \alpha) u(Y)-(Y \alpha) u(X)\}$ $+4 c \alpha\{(X h) u(Y)-(Y h) u(X)\}+2 c \alpha(2 h+\alpha) d u(X, Y)=0$.

Putting $X=\xi$ in this and taking account of Remark 1 and Lemma 2 , we find

$$
(2 h+\alpha) d u(\xi, X)=0 .
$$

Now, suppose that $\Omega_{1}=\left\{p \in \Omega \mid d u(\xi, X)_{p} \neq 0\right\}$ and $\Omega_{1} \neq \varnothing$. Then we have $2 h+\alpha=0$ and hence $h \alpha=-\frac{1}{2} \alpha^{2}$ on $\Omega_{1}$. Therefore (5.2) implies $\nabla \alpha=0$ and hence $\nabla h=0$ on $\Omega_{1}$. Accordingly $\nabla \beta=0$ because of (4.17) and Remark 1. Using these facts, (3.11) turns out to be

$$
3 A^{2} U-2 h A U+(\alpha h-\beta-c) U=0,
$$

which together with Lemma 1 and (4.8) gives $\alpha^{2}=h \alpha-2 c$ and consequently $3 \alpha^{2}=-4 c$ because we have already $h \alpha=-\frac{1}{2} \alpha^{2}$ on $\Omega_{1}$. Hence we see that $c<0$ on $\Omega_{1}$. By the way, (4.8) implies $2 \beta+\alpha^{2}=2 c$ on $\Omega_{1}$. It is contradictory by virtue of $c<0$. Therefore we have $\Omega_{1}=\varnothing$ and thus $d u(\xi, X)=0$ on $\Omega$ because of (5.3). From this fact, we have

$$
g\left(\nabla_{\xi} U, X\right)+g\left(\nabla_{X} \xi, U\right)=0 .
$$


which together with (2.9) and (2.12) implies that

$$
3 \phi A U+\alpha A \xi-\beta \xi+\phi \nabla \alpha+\mu A W=0
$$

or, using (2.6), (3.4) and Lemma $1,(3 c+h \alpha) \mu W+\phi \nabla \alpha=0$. Thus, it follows that

$$
\alpha \nabla \alpha=(\alpha h+3 c) U .
$$

From this and (5.2) we verify that

(5.5) $\alpha^{3}\left(c-\alpha^{2}\right) \nabla h=\left(2 c \alpha^{4}-h^{2} \alpha^{4}+c h \alpha^{3}+c \alpha^{2} h^{2}-5 c^{2} \alpha h+6 c^{3}\right) U$.

On the other hand, we have from (3.11)

$$
3 A^{2} U-2 h A U+A \nabla \alpha+\frac{1}{2} \nabla \beta-h \nabla \alpha+(\alpha h-\beta-c) U=0
$$

by virtue of Lemma 2 , which together with (5.1), (5.4) and Lemma 1 implies that

$$
\alpha^{2} \nabla h=\left(\alpha h^{2}+\frac{c^{2}}{\alpha}+c h\right) U
$$

Combining this to (5.5), we obtain

$$
\alpha^{4}+h \alpha^{3}+\frac{c}{2} \alpha^{2}+2 \operatorname{ch} \alpha+\frac{5}{2} c^{2}=0 .
$$

Differentiation this gives

$$
\left(4 \alpha^{3}+3 \alpha^{3} h+c \alpha+2 c h\right) \alpha \nabla \alpha+\left(\alpha^{2}+2 c\right) \alpha^{2} \nabla h=0 .
$$

which together with (5.4), (5.6) and (5.7) yields

$$
12 \alpha^{4}+9 \alpha^{2}(h \alpha)-4(\alpha h)^{2}-2 c(h \alpha)+4 c \alpha^{2}+2 c^{2}=0 .
$$

If we combine this to $(5.7)$, then we get

$$
\begin{gathered}
4\left(\alpha^{2}+\frac{c}{2} \alpha^{2}+\frac{5}{2} c^{2}\right)^{2}+\left(9 \alpha^{2}-2 c\right)\left(\alpha^{2}+2 c\right)\left(\alpha^{4}+\frac{c}{2} \alpha^{2}+\frac{5}{2} c\right) \\
=\left(\alpha^{2}+2 c\right)^{2}\left(12 \alpha^{4}+4 c \alpha^{2}+2 c^{2}\right),
\end{gathered}
$$

or equivalently $2 \alpha^{8}+55 c \alpha^{6}+\cdots+7 c^{4}=0$. Thus, $\alpha$ is a root of the algebraic equation with constant coefficient. So we verify that $\alpha$ is a constant on $\Omega$. Accordingly we have $\alpha h+3 c=0$ because of (5.4) and hence $h=$ constant. with the aid of Remark 1 . Consequently we see, using (5.6), that $(\alpha h)^{2}+c(\alpha h)+c^{2}=0$ and hence $c=0$ since $\alpha h+3 c=0$. It is contradictory. Thus, $\Omega=\varnothing$, namely $A \xi=\alpha \xi$ on $M$. This completes the proof. 


\section{References}

1. J. Berndt, Real hypersurfaces with constant principal curvatures in complex hyperbolic spaces, J. Reine Angew. Math. 395(1989), 132-141.

2. J. Berndt and H. Tamaru, Cohomogeneity one actions on noncompact symmetric spaces of rank one, Trans. Amer. Math. Soc. 359(2007), 3425-3438.

3. J. T. Cho and U-H. Ki, Jacobi operators on real hypersurfaces of a complex projective space, Tsukuba J. Math. 22(1998), 145-156.

4. J. T. Cho and U-H. Ki, Real hypersurfaces of a complex projective space in terms of Jacobi operators, Acta Math. Hungar. 80(1998), 155-167.

5. J. T. Cho and U-H. Ki, Real hypersurfaces in a complex space form with Reeb flow symmetric Jacobi operator, Canadian Math. Bull. 51(2008), 359-371.

6. U-H. Ki, S. J. Kim and S.-B. Lee, The structure Jacobi operator on real hypersurfaces in a nonflat complex space form, Bull. Korean Math. Soc. 42(2005), 337-358.

7. U-H. Ki and H. Kurihara, Real hypersurfaces and $\xi$-parallel structure Jacobi operators in complex space forms, J. Academy Scien. Korea 48(2009), 53-78.

8. U-H. Ki and H. Kurihara, Real hypersurfaces with cyclic-parallel structure Jacobi operators in a nonflat complex space form, Bull. Aust. Math. Soc. 81(2010), 260-273.

9. U-H. Ki and H. Kurihara, S. Nagai and R. Takagi, Characterizations of real hypersurfaces of type A in a complex space form in terms of the structure Jacobi operator, Toyama Math. J. 32(2009), 5-23.

10. U-H. Ki, H. Kurihara and R. Takagi, Jacobi operators along the structure flow on real hypersurfaces in a nonflat complex space form, Tsukuba J. Math. 33(2009), 39-56.

11. U-H. Ki and S. Nagai, The Ricci tensor and structure Jacobi operator of real hypersurfaces in a complex projective space, J. Geom. 94(2009), 123-142.

12. U-H. Ki, J. D. Pérez, F. G. Santos and Y. J. Suh, Real hypersurfaces in complex space forms with $\xi$-parallel Ricci tensor and structure Jacobi operator, J. Korean Math. Soc. 44(2007), 307-326.

13. U-H. Ki and Y. J. Suh, On real hypersurfaces of a complex space form, Math. J. Okayama Univ. 32(1990), 207-221.

14. N-G. Kim and U-H. Ki, $\xi$-parallel structure Jacobi operators of real hypersurfaces in a nonflat complex space form, Honam Math. J. 28(2006), 573-589.

15. R. Niebergall and P. J. Ryan, Real hypersurfaces in complex space forms in Tight and Taut Submanifolds, (eds. T. E. Cecil and S. S. Chern)(Cambridge University Press, Cambridge,1998,) 233-305.

16. M. Ortega, J. D. Pérez and F. G. Santos, Non-existence of real hypersurfaces with parallel structure Jacobi operator in nonflat complex space forms, Rocky Mountain J. 36(2006), 1603-1613.

17. J. D. Pérez, F. G. Santos and Y. J. Suh, Real hypersurfaces in complex projective spaces whose structure Jacobi operator is $D$-parallel, Bull. Belg. Math. Soc. Simon Stevin 13(2006), 459-469.

18. R. Takagi, On homogeneous real hypersurfaces in a complex projective space, Osaka J. Math. 19(1973), 495-506. 
19. R. Takagi, Real hypersurfaces in a complex projective space with constant principal curvatures I, II, J. Math. Soc. Japan 15 (1975), 43-53, 507-516.

\section{Soo Jin Kim}

Department of Mathematics,

Chosun University,

Kwangju, 501-759, S.Korea.

E-mail: sj-kim@chosun.kr 